\title{
GLOBAL PROPERTIES OF HIGH-ENERGY EMISSION FROM GAMMA-RAY BURSTS
}

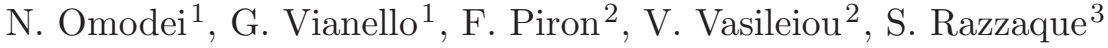 \\ and the Fermi Large Area Telescope collaboration
}

\begin{abstract}
In three years of observations the Large Area Telescope (LAT) on board the Fermi Gamma Ray Space Telescope has observed highenergy $\gamma$-ray emission from 35 gamma-ray bursts (GRBs). The first Fermi-LAT catalog of GRBs is in preparation within the Fermi LAT collaboration and will provide a systematic study of high-energy emission from GRBs. In this paper we present some of the main results, briefly discussing durations, energetics, time-resolved and time integrated LAT spectral analysis. We also discuss characteristics of LATdetected emission such as its delayed onset and longer duration compared to emission detected by the GBM, and its power-law temporal decay at late times.
\end{abstract}

\section{Introduction}

The Fermi observatory was placed into orbit on 2008 June 11. It provides unprecedented breadth of energy coverage and sensitivity for advancing knowledge of GRB properties at high energies. It has two instruments: the Gamma-ray Burst Monitor (GBM; Meegan et al. 2009), comprised of twelve sodium iodide (NaI) and two bismuth germanate (BGO) detectors sensitive in the $8 \mathrm{keV}-1 \mathrm{MeV}$ and $150 \mathrm{keV}-40 \mathrm{MeV}$ respectively, and the Large Area Telescope (LAT; Atwood et al. 2009), a pair conversion telescope sensitive to $\gamma$ rays in the energy range from $\sim 20 \mathrm{MeV}$ to $\gtrsim 300 \mathrm{GeV}$. The wide field of view $(\sim 2.4$ sr at $1 \mathrm{GeV})$ of the LAT, its continuous observations in scanning mode, its broad energy range, its large effective area $\left(\sim 6500 \mathrm{~cm}^{2}\right.$ on axis at usin $\left.>1 \mathrm{GeV}\right)$, its low dead time per event $(\sim 27 \mu \mathrm{s})$, its efficient background rejection, and its good angular resolution

\footnotetext{
${ }^{1}$ Stanford University/KIPAC

${ }^{2}$ CNRS/IN2P3/LUPM

${ }^{3}$ GMU/NRL
} 
$(\sim 0.8$ at $1 \mathrm{GeV})$ are vastly improved in comparison with those of EGRET. As a result, the LAT provides more GRB detections, higher statistics per detection, and more accurate localizations $\left(\lesssim 1^{\circ}\right)$.

\section{Properties of Gamma-Ray Burst emission at high energy}

We have analyzed 3 years of Fermi LAT data using Pass 6 v3 Transient-event class above $100 \mathrm{MeV}$, significantly detecting 28 GRBs. We have also detected 7 additional GRBs using the "LAT Low Energy" (LLE) event class (Pelassa et al. 2010) which increases the effective area between $\sim 20 \mathrm{MeV}-\sim 1 \mathrm{GeV}$. While the rate of LAT GRBs is a small fraction of the rate of GRBs detected by the FermiGBM (Goldstein et al. 2012; Paciesas et al. 2012), there are unique features that emerge only at high energies. First we compute the onset time $\left(\mathrm{T}_{05}\right)$ and the duration $\left(\mathrm{T}_{90}\right)$ of the high-energy emission, and we compare our measurements with the durations reported in the GBM GRB catalog (Paciesas et al. 2012). In the left (right) panel of Figure 1 the $>100 \mathrm{MeV}$ LAT $\mathrm{T}_{05}\left(\mathrm{~T}_{90}\right)$ is compared to the GBM results (in the $50 \mathrm{keV}-300 \mathrm{keV}$ energy band).
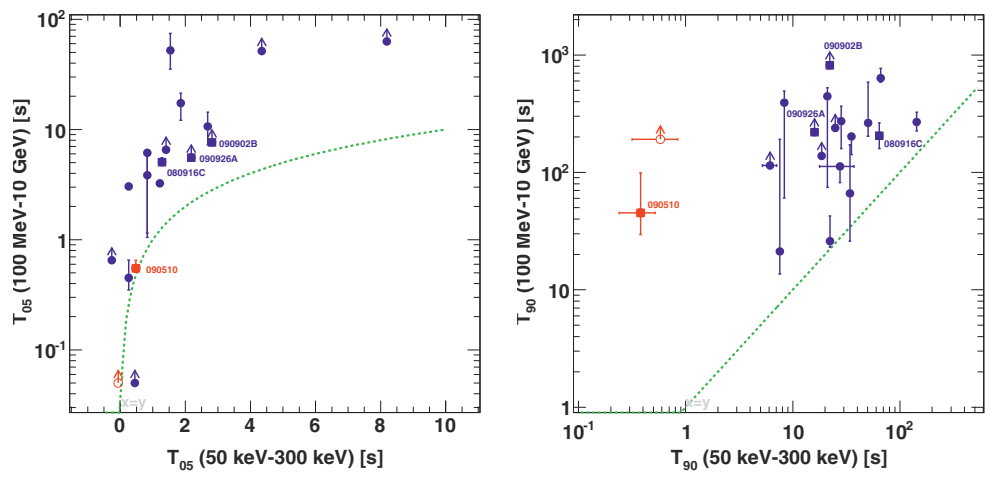

Fig. 1. Left: comparison between the $>100 \mathrm{MeV} \mathrm{T}_{05}$ as measured using the LAT Transient class events and the $50 \mathrm{keV}-300 \mathrm{keV} \mathrm{T}_{05}$ as measured by the GBM. Right: $\mathrm{T}_{90}$. GBM data from Paciesas et al. (2012). Long (short) GRBs with filled blue (empty red) markers. Squares markers highlight the 4 brightest bursts (labeled).

We use the maximum likelihood technique implemented in the Fermi LAT ScienceTools to perform the spectral analysis from which we derive the fluences of the LAT GRBs. The left panel of Figure 2 shows the fluence measured by the LAT versus the fluence measured by the GBM. The bulk of the LAT GRB population, primarily composed of long GRBs, has a ratio of high- $(100 \mathrm{MeV}-10 \mathrm{GeV})$ to low-energy $(10 \mathrm{keV}-1 \mathrm{MeV})$ fluence $\lesssim 20 \%$. It is interesting to note that short LAT-detected bursts (red markers) have a greater ratio of high- to low-energy fluence than the bulk of the long-GRB population (blue markers). In this figure, 
we can see four hyper-fluent LAT bursts, GRBs 080916C, 090510, 090902B, and 090926A, having a more intense emission in the LAT energy range compared to the rest of the GRB population. It is worth examining whether the four brightest LAT bursts appear bright because they are systematically closer to us compared to the rest of the GRB population. To verify this, we calculate the $\mathrm{E}_{\text {iso }}$ in the $1 \mathrm{keV}-10 \mathrm{MeV}$ rest frame energy range (shown as a function of the redshift in the right panel Fig. 2). The energy range matches that of previous works (Butler et al. 2007 for Swift bursts and Goldstein et al. 2012 for GBM bursts), allowing direct comparisons of $\mathrm{E}_{\mathrm{iso}}$. We find that the four brightest bursts also have the highest $\mathrm{E}_{\text {iso }}$ in their respective (long and short) categories.

Using LAT detections of GRBs, it has been discovered that extra powerlaw components are more common in GRBs compared to what was previously thought. More importantly, even if the high-energy emission can last longer than the usual keV-to-MeV emission, in some cases (GRBs 090510, 090902B, 090926A) it contributes significantly during the prompt phase. These two considerations suggest that the total energy budget at high energies can be an important fraction of the total energy reservoir.
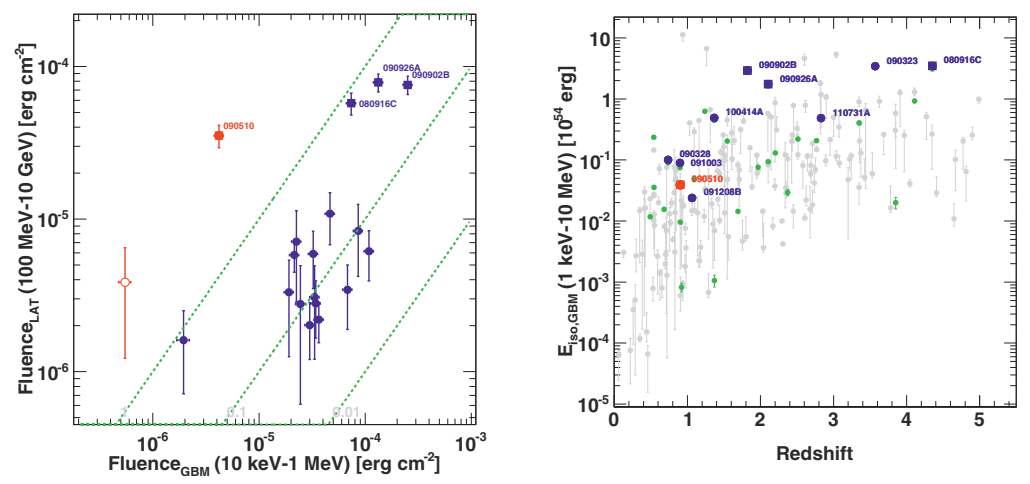

Fig. 2. Left: fluence measured by the LAT versus the fluence measured by the GBM. The three dashed lines denote the $100 \%, 10 \%$ and $1 \%$ fluence ratios. Colored symbols follow the convention of Figure 1. Right: isotropic energy in the $1 \mathrm{keV}-10 \mathrm{MeV}$ energy range of LAT-detected GRBs (blue/red symbols) compared with Swift GRBs (Butler et al. 2007) (grey dots) and GBM GRBs (Goldstein et al. 2012) (green).

To study the temporal decay of the extended emission detected by the LAT, we apply a time-resolved spectral analysis, and we compute the isotropic equivalent luminosity (as defined in Ghisellini et al. 2010) as a function of time, since the GBM trigger time. We then investigate the temporal decay by fitting the lightcurve with a simple power law or with a broken power law. Observations before the peak flux are excluded from the fit. In the left panel of Figure 3 we show all the detected long-lasting emissions, while in the right panel, we isolate the three GRBs (090510, 

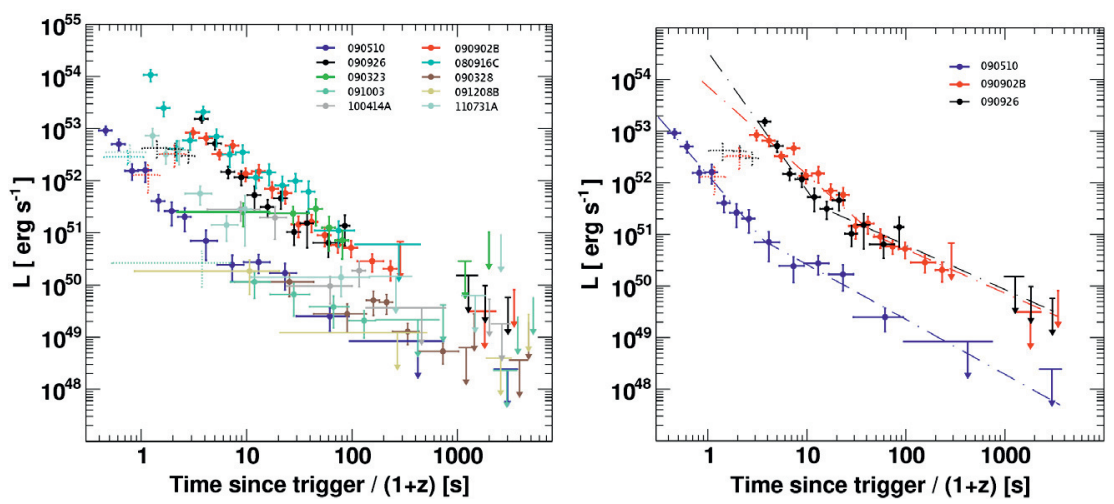

Fig. 3. Left: the decay of the luminosity $\mathrm{L}$ with time measured in the rest frame for all the GRBs with detected extended emission. Right: same quantity, but for 3 GRBs with a significant time break detected. Dashed-dotted lines are the best fits of the broken power law model to each GRB, while dashed crosses are the luminosities before the peak times, which have not been used in the fits.

090902B and 090926A) with a significant break detected in the lightcurve (chance probability smaller than $10^{-3}$ ).

\section{Discussion}

In this study, we have presented some of the results that will be published in a LAT collaboration paper characterizing the high-energy emission of Fermi-LAT detected GRBs. Here we briefly discuss few important points.

The origin of the delayed onset of the LAT emission is poorly understood, but a possible interpretation is based on the early afterglow model for the temporallyextended LAT emission (De Pasquale et al. 2010; Ghisellini et al. 2010; Kumar \& Barniol Duran 2009; Razzaque 2010). The bolometric flux from a coasting fireball increases as $\propto t^{2}$ (Sari 1997) before it decelerates and enters a self-similar phase (Blandford \& McKee 1976; Rees \& Meszaros 1994). The time required for the flux to increase and be detected by the LAT corresponds to the delay time in this scenario. The flux of LAT-detected emission at late times decays rather smoothly and can generally be fitted with a power law $F_{\nu} \propto t^{-\alpha_{L}}$. For three bright bursts (GRBs 090510, 090902B and 090926A), a broken power law fits the LAT data better than a single power law with the initial flux decay steeper than the later one, which is always close to $\alpha_{L}=1$. In the context of the external shock, the bolometric flux decays as $\propto t^{-\alpha}$, with $\alpha=1$ and $\alpha=10 / 7$ for an adiabatic and a radiative fireball in a constant density environment (Ghisellini et al. 2010; Katz \& Piran 1997; Sari 1997), respectively. A simple interpretation of $\alpha_{L} \approx 1$ flux decay-index for most LAT bursts suggests that the $\gtrsim 100 \mathrm{MeV}$ emission in 
the long-lasting phase is more likely from an adiabatic fireball (De Pasquale et al. 2010; Kumar \& Barniol Duran 2009; Razzaque 2010) rather than from a radiative fireball, as Ghisellini et al. (2010) had suggested. The observed break in the decay rate of the extended emission might be due to a transition from the prompt (internal shocks) to afterglow (external shock) emission.

Spectral analysis of bright bursts shows that the GRBs in which we detect with high significance a break in the lightcurves (GRBs 090510, 090902B and 090926A), also require an additional extra component (Abdo et al. 2009a,b; Ackermann et al. 2010, 2011; Giuliani et al. 2010). In these cases, the high-energy photon index of the Band function $(\beta)$, is systematically softer (and more variable) than the photon index of the high energy extra component, indicating again a possible dichotomy between the prompt and the early afterglow.

Finally, the fluence of LAT GRBs (see Fig. 2) provides hints of two classes: a hyper-fluent class currently with four members (GRBs 080916C, 090510, 090902B, and 090926A); and a larger class with a lower typical fluence. The bolometric isotropic equivalent energy $E_{\text {iso }}$ (see Fig. 2, right panel) is also higher for LAT bursts, suggesting that, in agreement with Cenko et al. (2011) and Racusin et al. (2011), LAT bursts possibly comprise the most energetic sub-sample of GRBs.

The Fermi LAT Collaboration acknowledges support from a number of agencies and institutes for both development and the operation of the LAT as well as scientific data analysis. These include NASA and DOE in the United States, CEA/Irfu and IN2P3/CNRS in France, ASI and INFN in Italy, MEXT, KEK, and JAXA in Japan, and the K.A. Wallenberg Foundation, the Swedish Research Council and the National Space Board in Sweden. Additional support from INAF in Italy and CNES in France for science analysis during the operations phase is also gratefully acknowledged.

\section{References}

Abdo, A.A., Ackermann, M., Ajello, M., et al., 2009a, ApJ 706, L138

Abdo, A.A., Ackermann, M., Ajello, M., et al., 2009b, Nature, 462, 331

Ackermann, M., Ajello, M., Asano, K., et al., 2011, ApJ, 729, 114

Ackermann, M., Asano, K., Atwood, W.B., et al., 2010, ApJ, 716, 1178

Atwood, W.B., Abdo, A.A., Ackermann, M., et al., 2009, ApJ, 697, 1071

Blandford, R.D., \& McKee, C.F., 1976, BAAS, Vol. 8, 539

Butler, N.R., Kocevski, D., Bloom, J.S., \& Curtis, J.L., 2007, ApJ, 671, 656

Cenko, S.B., Frail, D.A., Harrison, F.A., et al., 2011, ApJ, 732, 29

De Pasquale, M., Schady, P., Kuin, N.P.M., et al., 2010, ApJ, 709, L146

Ghisellini, G., Ghirlanda, G., Nava, L., \& Celotti, A., 2010, MNRAS, 403, 926

Giuliani, A., Fuschino, F., Vianello, G., et al., 2010, ApJ, 708, L84

Goldstein, A., Burgess, J.M., Preece, R.D., et al., 2012, ApJS, 199, 19

Katz, J.I., \& Piran, T., 1997, ApJ, 490, 772

Kumar, P., \& Barniol Duran, R., 2009, MNRAS, 400, L75 
Meegan, C., Lichti, G., Bhat, P.N., et al., 2009, ApJ, 702, 791

Paciesas, W.S., Meegan, C.A., von Kienlin, A., et al., 2012, ApJS, 199, 18

Pelassa, V., Preece, R., Piron, F., et al., 2010 [ArXiv e-prints]

Racusin, J.L., Oates, S.R., Schady, P., et al., 2011, ApJ, 738, 138

Razzaque, S., 2010, ApJ, 724, L109

Rees, M.J., \& Meszaros, P., 1994, ApJ, 430, L93

Sari, R., 1997, ApJ, 489, L37 